\title{
Need of Improvement in Timing of Prophylactic Antibiotic in Elective Surgery
}

\author{
Shah JN, ${ }^{1}$ Maharian SB, ${ }^{1}$ Piya ${ }^{2},{ }^{2}$ Shrestha $A,{ }^{2}$ Shrestha $K_{,}{ }^{2}$ Basnet NB, ${ }^{2}$ Gurung $\mathrm{HM}^{2}$ \\ 'Department of surgery, ${ }^{2}$ Department of Anesthesia, Patan Hospital, Patan Academy of Health Science (PAHS), Lalitpur, Nepal.
}

\section{ABSTRACT}

Introduction: Infections in surgery are major concern of morbidity, mortality, and costs. Timely antibiotic prophylaxis before incision ensures optimum concentration of AP in blood and tissues to prevent surgical site infections. However, proper timing of AP remains problematic as reported by various studies, though none so far from Nepal. Aim of this prospective observational study was to assess and address the issues for improvements in timing of AP.

Methods: The pattern of antibiotic prophylaxis were prospectively collected in respect to time of induction, and incision time were recorded on predesigned 'AP form'. The study included all the elective major cases who received AP of intravenous Cefazoline $1 \mathrm{~g}$ as per our existing protocol. The emergency surgeries and obstetric cases were excluded from the study.

Results: There were 125 cases of which $89 \%$ received AP before incision (63\% within 5 minutes before incision), while $11 \%$ had AP after the incision and $1 \%$ within the recommended time period of 2 hour to 30 minutes before incision.

Conclusions: Current practice of antibiotic prophylaxis (AP) needs improvement as per standard guidelines of AP within 2 hour to 30 before incision.

Keywords: antibiotic, infection, prophylaxis, surgery

\section{INTRODUCTION}

Surgical site infections (SSIs) adversely affect the quality of life by prolonged hospital stay, readmission, excess cost, increased morbidity and mortality. ${ }^{1-3}$

The effectiveness of antibiotic prophylaxis (AP) before skin incision in prevention of SSIs is well established. Nearly $50 \%$ of SSIs can be prevented with proper administration of AP on time is critical as studies and guidelines state that AP should be given within 60 to 30 minutes before incision to achieve therapeutic levels. ${ }^{4-6}$

Despite various guidelines the timing AP is often not optimal and frequently given only seconds prior to the surgical incision. ${ }^{6-10}$

We undertook this prospective study to evaluate and address the issues for improvements in timing of AP at our institution. There is lack of published data on 
Shah et al. Need of Improvement in Timing of Prophylactic Antibiotic in Elective Surgery

existing practices in major institutions. Also, there is no published recommendation and guidelines of AP from concerned societies or academic institutions. We hope this study will provide insight and prompt institutions and academia to look into the issue of AP, and hopefully come up with necessary effective measures to ensure proper use of AP.

\section{METHODS}

A prospective, cross-sectional study was conducted in the department of surgery in Patan Hospital in the month of October, 2009 for one month or for at least 100 consecutive patients. The study was discussed within the department of surgery and anesthesia and endorsed by hospital authority. All surgical patients gave written informed consent for surgery. Verbal consent was taken for the collection of data on use of antibiotic prophylaxis.

The date, type of surgery, time of induction, AP administration and incision time was recorded on predesigned 'AP form'. Forms were kept in each operation room. Members of anesthetic team were requested to fill in the forms.

The study included all the elective major cases from general surgery, urology, orthopedic and gynecology procedures that received AP as per existing protocol. Floor nurse were not aware of the study to decrease biasness as currently they are designated to administer the AP. Intravenous Cefazoline $1 \mathrm{~g}$ was administered in all the major surgeries unless differently demanded by situation. This study was discussed within the department of surgery and anesthesia and endorsed by hospital authority. All surgical patients gave written informed consent for surgery. Verbal consent was taken for the collection of data of antibiotic prophylaxis.

For ease of clinical application the timing of AP is divided in different period in reference to the time of incision.

Definition of AP based on timing

Early - during two to 24 hours before the surgical incision

Preoperative- during the two hours before the incision

Perioperative- during the three hours after the incision

Postoperative- more than three but less than 24 hours after the incision

The emergency surgeries and obstetric cases were excluded from the study. Timing of administration was analyzed in intervals before and after incision. Microsoft excel 2007 was used for data analysis.

\section{RESULTS}

There were total of 125 cases eligible for analysis. Male patients were 55 (44\%). Majority (92\%) of our patients were adult 18 years or above because we take only simple pediatric cases (Figure 1). General surgical procedures $(45 \%)$ were more in numbers as we have four operating days every week. Urology is combined with the general surgery operating days (Table 1). Orthopedic and gynecology procedures have one day per week. Out of 125 cases, 101 (89\%) received AP before incision, majority within five minutes $(n=64)$, while $24(11 \%)$ had AP after the incision (Table 2$)$. Only $1(1 \%)$ AP was administered in the recommended period of within two hour to 30 minutes before incision.

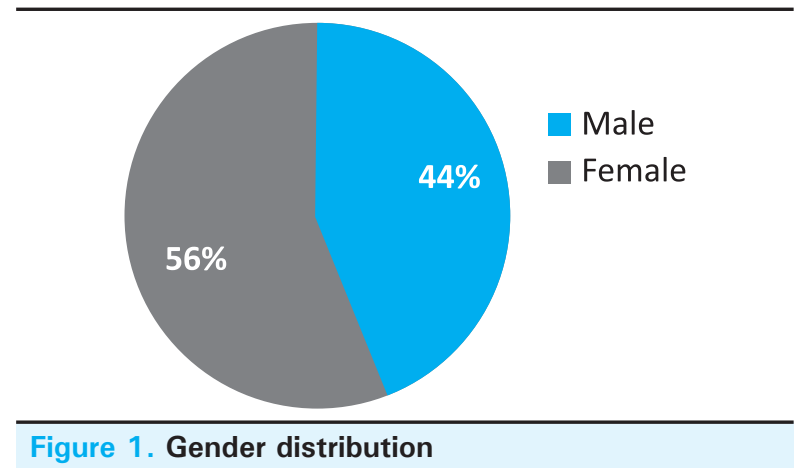

Figure 1. Gender distribution

Table 1. Types of surgery during study period (Total 125)

\begin{tabular}{ccc}
\hline Types of surgery & $\mathbf{n}$ & $\%$ \\
\hline General & 57 & 45 \\
- & 20 & 16 \\
Urology & 26 & 21 \\
Gynecology & 22 & 18 \\
\hline Total & 125 & 100 \\
\hline
\end{tabular}

Table 2. Timing (minute) of antibiotic prophylaxis (AP) in 125 elective major surgeries

\begin{tabular}{ccc}
\hline AP before incision $(\mathbf{n}=\mathbf{1 0 1 ;} \mathbf{8 9} \%)$ & $\mathbf{n}$ & $\%$ \\
\hline$<5$ minutes & 64 & 63 \\
$6-15$ & 25 & 25 \\
$16-30$ & 11 & 11 \\
$31-120$ & 1 & 1 \\
\hline AP after incision $(\mathbf{n}=\mathbf{2 4 ;} \mathbf{1 1} \%)$ & & \\
\hline$<5$ minutes & 11 & 46 \\
$6-15$ & 7 & 29 \\
$>15$ & 6 & 25 \\
\hline
\end{tabular}

\section{DISCUSSION}

Surgical site infections cause significant morbidity, mortality and add to the cost of care. Aseptic techniques alone do not eliminate bacteria, and $S$. aureus are often 
Shah et al. Need of Improvement in Timing of Prophylactic Antibiotic in Elective Surgery

found at surgical sites. Antimicrobial treatment begun prior to contamination, referred to as prophylaxis, is an important adjunct to control bacterial growth and SSIs.

AP is commonly administered at the induction of anesthesia, but the drugs are frequently given only seconds prior to the surgical incision which is inappropriate to achieve adequate tissue levels of drugs and effective prophylaxis. ${ }^{10}$

In this study we found that out of $89 \%$ APs, $63 \%$ were administered within five minutes prior to incisions. And we hope that other major institutions are performing better, though we doubt, in view of lack of local guidelines on practices of AP.

Administration of antibiotics in the two hour period (ideally 30-60 minutes) before surgical incision is associated with the lowest risk of surgical wound infection. ${ }^{4}$

In a randomized control trial of 2847 patients with 'clean, clean-contaminated' surgeries, AP administered within two hours before surgical incision was associated with the lowest risk of wound infections. ${ }^{4}$

Most of the research has been targeted in determining which antibiotic is most effective and little emphasis on appropriate timing of AP. In this study we have focused on important aspect of timing of AP.

A number of guidelines are available for selection, timing, and duration of AP in various types of surgeries. ${ }^{11}$ However, there is often lack of effective system to ensure this simple task, leading to patient injury, mortality, and increased health care costs.

Health care-associated infection (HAI) is unavoidable complications of life-saving interventions but often it is the failure of system due to inadequate resourcing. This is not only an ethical problem involving patients' rights; it is also the obligation of health care institutions as well as individual health care provider.

Failure in the proper timing of AP despite having knowledge of guidelines includes workflow and role perception. Other factors are low priority, inconvenience and organizational communication. Addressing individual values, professional, and organizational conflicts is necessary to achieve optimal practice. ${ }^{12}$

Guidelines, regulations, and recommendations are modified frequently, and the Internet Web sites are a valuable resource for the up-to-date information on relevant issues of infection prevention and control. CDC (Centers for Disease Control and Prevention), APIC (Association for Professionals in Infection Control and Epidemiology) and SHEA (Society for Healthcare Epidemiology of America) are some of the useful sites. ${ }^{13}$
Timely administration of AP before surgery continues to be a difficult challenge. Circulating floor nurses in operation theater (OT) play important role in administration of AP. The anesthesiologists are not ordinarily responsible for AP even though they are closely involved in planning and outcome of surgery. When anesthesia department assumed responsibility for timing and administration of AP with the goal of ensuring that patients receive antibiotic prophylaxis within 60 minutes before incision, there was steady increase in compliance resulting in significant improvement from $69 \%$ to $92 \%$ patients getting timely AP a year later. ${ }^{14}$

Incidence of $\mathrm{SSI}$ in relation to timing of $\mathrm{AP}$ in a consecutive series of 3836 surgical procedures found that drug given within one hour to half an hour before incision was most effective. ${ }^{15}$

In national retrospective cohort study with medical record review from 2965 US hospitals involving random sample of 34133 cases revealed that only $55.7 \%$ of patients received AP within one hour before incision. ${ }^{11}$

In a large prospective cohort study with single drug (Cefuroxime) analyzed the data by 15 minute intervals in the 74 minutes preceding incision found that 59 to 30 minutes interval before incision was most effective. ${ }^{15}$

SSIs is significantly increased when AP is given after incision whereas prolonged prophylaxis after the end of surgery had no contribution in a multi-centric study of 1922 patients with elective total hip arthroplasty. The study concluded that intervention programs should focus on timely administration of AP. In our study $11 \%$ of AP were given after the incision. ${ }^{16}$

In orthopedic cases, in a randomized, double-blind, placebo-controlled trial in 908 patients concluded that administration of prophylactic antibiotics just before tourniquet release was not inferior to standard antibiotic prophylaxis. ${ }^{17}$

When surgery is prolonged the antibiotic tissue levels decreases during later phase and re-administering antibiotic may be given if surgery prolongs for more than four hours. Similarly, in special circumstances with different pharmacokinetics and drug delivery (like rapid increase in the volume at the initiation of cardiopulmonary bypass or decreased circulation to the surgical site in patients with diabetes or arteriosclerosis) the timing of AP may not be suitable for as close to incision time as possible. ${ }^{18}$

Some of the recommendations of Centers for Disease Control and Prevention (CDC) include timely intravenous administration of AP in indicated cases before incision for effective concentration of drug against most common pathogens causing SSI. Therapeutic levels drug 
Shah et al. Need of Improvement in Timing of Prophylactic Antibiotic in Elective Surgery

should be maintained in serum and tissues throughout the operation and until, at most, a few hours after the incision is closed. ${ }^{19}$

Evidence-based practice from the perspective of individual, team, and organizational levels need to be considered $^{12,20-2}$ like individual routines; responsibility for AP administration; and workflow changes to ensure proper timing of AP.

The 'AP form recording the timing' of antibiotic administration may be an effective tool to decrease the inappropriate use of antibiotics as shown in a retrospective study before and after the introduction of such 'form'. ${ }^{23}$

Auditing and raising the awareness of surgeons and nurses about the regimen, importance of the timing of AP with better documentation are helpful in ensuring intended administration of AP. ${ }^{24}$

After this observational study, we have discussed with anesthesiologist and operating room nurse in-charge, to change the current practice of floor nurse being solely responsible for AP to let anesthesiologist assume overall responsibility in collaboration with floor nurse. Surgical team will, periodically audit and provide monthly feed back to increase awareness of importance of AP timing to the all involved in care of patients with hope to have desired improvement in timing of AP at par with standard guidelines.

\section{CONCLUSIONS}

Our result shows we are not following the standard guidelines of antibiotic prophylaxis (AP) administration within 60-30 minutes before incision. We need to improve our current practice of AP by team work among nurses, anaesthetics and surgeons.

\section{ACKNOWLEDGMENTS}

We would like to acknowledge the invaluable support of anaesthetic team for their help in completing the performa.

\section{REFERENCES}

1. Whitehouse JD, Friedman ND, Kirkland KB, Richardson WJ, Sexton DJ. The impact of surgical-site infections following orthopedic surgery at a community hospital and a university hospital: adverse quality of life, excess length of stay, and extra cost. Infect Control Hosp Epidemiol. 2002;23:183-9.

2. Coello R, Charlett A, Wilson J, Ward V, Pearson A, Borriello P. Adverse impact of surgical site infections in English hospitals. J Hosp Infect. 2005;60:93-103.

3. Perencevich EN, Sands KE, Cosgrove SE, Guadagnoli E, Meara E, Platt R. Health and economic impact of surgical site infections diagnosed after hospital discharge. Emerg Infect Dis. 2003;9:196-203.

4. Classen DC, Evans RS, Pestotnik SL, Horn SD, Menlove RL, Burke JP. The timing of prophylactic administration of antibiotics and the risk of surgical-wound infection. N Engl J Med. 1992;326:281-6

5. Bratzler DW, Houck PM. Antimicrobial prophylaxis for surgery: an advisory statement from the National Surgical Infection Prevention Project. Am J Surg. 2005;189:395-404.

6. Mangram AJ, Horan TC, Pearson ML, et al. Guideline for prevention of surgical site infection, 1999. Hospital Infection Control Practices Advisory Committee. Infect Control Hosp Epidemiol. 1999;20:250-78.

7. Gilbert DN, Moellering RC, Sande MA. The Sanford Guide to Antimicrobial Therapy, 2003. 33rd ed. Hyde Park, Vt: Antimicrobial Therapy Inc; 2003: p. 123-4.

8. Silver A, Eichorn A, Kral J, et al. Timeliness and use of antibiotic prophylaxis in selected inpatient surgical procedures. Am J Surg. 1996;171:548-52.
9. Gorecki P, Schein M, Rucinski JC, Wise L. Antibiotic administration in patients undergoing common surgical procedures in a community teaching hospital: the chaos continues. World J Surg. 1999;23:429-33.

10. Burke JP. Maximizing appropriate antibiotic prophylaxis for surgical patients: an update from LDS Hospital, Salt Lake City. Clin Infect Dis. 2001;33(suppl 2):S78-S83.

11. DW Bratzler et al, “Use of antimicrobial prophylaxis for major surgery: Baseline results from the National Surgical Infection Prevention Project." Archives of Surgery. 2005;140:174-82.

12. Tan JA, Naik VN, Lingard L. Exploring obstacles to proper timing of prophylactic antibiotics for surgical site infections. Qual Saf Health Care. 2006;15:32-8.

13. Johnson LE, Reyes K, Zervos MJ. Resources for Infection Prevention and Control on the World Wide Web. Clin Infect Dis. 2009;48(11):1585-95.

14. Michael O'Reilly, AkkeNeel Talsma, Sharon VanRiper, Sachin Kheterpal, Richard Burney. An anesthesia information system designed to provide physician-specific feedback improves timely administration of prophylactic antibiotics. Anesth Analg. 2006;103:908-12.

15. Walter P. Weber, WR Marti, M Zwahlen, et al. The Timing of Surgical Antimicrobial Prophylaxis. Ann of Surg 2008;247(6):918-26.

16. Marjo E. E. van Kasteren, Judith Mannien, Alewijn Ott, Bart-Jan Kullberg, Annette S. de Boer, and Inge C. Gyssens. Infections following total hip arthroplasty: timely administration is the most important factor. Clinical Infectious Diseases. 2007;44(1 April):921-7. 
17. Soriano A, Bori G, García-Ramiro S, Martinez-Pastor JC, Miana T, Codina C, Maculé F, Basora M, Martínez JA, Riba J, Suso S, Mensa J. Timing of antibiotic prophylaxis for primary total knee arthroplasty performed during ischemia. Clinical Infectious Diseases. 2008;46:1009-14

18. Garey KW, Dao T, Chen $\mathrm{H}$, et al. Timing of vancomycin prophylaxis for cardiac surgery patients and the risk of surgical site infections. J Antimicrob Chemother. 2206;58:64550.

19. Mangram AJ, Horan TC, Pearson ML, Silver LC, Jarvis WR. Guideline for prevention of surgical site infection, 1999. Centers for disease control and prevention (CDC) hospital infection control practices advisory committee. Am J Infect Control. 1999;27(2):97-132.
20. Grol R, Grimshaw J. From best evidence to best practice: effective implementation of change in patients' care. Lancet. 2003;362:1225-30.

21. Pope C, van Royen P, Baker R. Qualitative methods in research on healthcare quality. Qual Saf Health Care. 2002;11:148-52.

22. Grol R. Personal paper: Beliefs and evidence in changing clinical practice. BMJ. 1997;315:418-21.

23. Pan SC, Sun HY, Lin JW, Lin C, Lai TS, Chang SC. Improvement in timing of antibiotic administration by using a prophylactic antibiotic record form. J Formos Med Assoc 2008;107(3):21824.

24. Raghuram Thonse, Muthyala Sreenivas, Kevin P Sherman. Timing of antibiotic prophylaxis in surgery for adult hip fracture. Ann R Coll Surg Engl. 2004;86:263-6. 